\title{
Mesoporous Biomaterials: a Lexicon and Structured Bibliography of Reviews
}

\section{Kennedy, Elizabeth}

2016-05-05

Kennedy , E , Canham , L \& Almeida Santos , H 2016 , ' Mesoporous Biomaterials: a Lexicon and Structured Bibliography of Reviews ' , Mesoporous Biomaterials , vol. 3 , no. 1 , pp. 1-14 . https://doi.org/10.1515/mesbi-2016-0001

http://hdl.handle.net/10138/163788

https://doi.org/10.1515/mesbi-2016-0001

cc_by_nd

publishedVersion

Downloaded from Helda, University of Helsinki institutional repository.

This is an electronic reprint of the original article.

This reprint may differ from the original in pagination and typographic detail.

Please cite the original version. 


\section{Mesoporous Biomaterials: a Lexicon and Structured Bibliography of Reviews}

DOI 10.1515/mesbi-2016-0001

Accessing mesoporous and nanostructured biomaterial information can be particularly time-consuming and problematic due to the interdisciplinary nature of the field. Relevant papers can be found across a broad range of journals and various key words are needed to comprehensively retrieve information. A keyword lexicon and themed bibliography of over 250 review articles from more than 100 different journals has therefore been assembled to aid students and researchers in this expanding field. Reviews dedicated to fabrication of mesoporous materials and some key characterisation techniques are grouped together. Reviews focussed on specific medical issues like biocompatibility and toxicological testing are also provided. Medical applications that have been the focus of reviews include drug delivery, cancer therapy, medical imaging, orthopaedics, tissue engineering, biofiltration, biosensing and bioanalysis. The following mesoporous materials also have had dedicated reviews on biomedical uses: silica, silicon, silicates, metallic biomaterials, metal organic frameworks, carbonaceous materials, calcium phosphates, titania, and alumina.

The academic literature, excluding textbook and textbook chapters, was searched using the lexicon shown in table 1 to capture the field, in conjunction with "review". Searching was conducted via Google, PubMed, Scopus, Google Scholar and Web of Science.

Reviews on mesoporous materials which focussed on non-medical applications were also excluded. Reviews based on nanoscale component structures with mesoporosity, like nanotubes, were included, whilst reviews solely on solid nanoparticles were not. The reviews found have been grouped as shown schematically in figure 1, depending on whether their emphasis is on fabrication, characterisation, medical testing or specific medical uses. General reviews which featured both medical and nonmedical applications have been included, but those focussed solely on non-medical applications have not. Reviews which discuss the applicability of various character- ization techniques to mesoporous materials in general, or specific mesoporous materials, were included. Most of the reviews that focus on one specific mesoporous material for one specific medical application were grouped within the medical application section, rather than the corresponding dedicated material section.

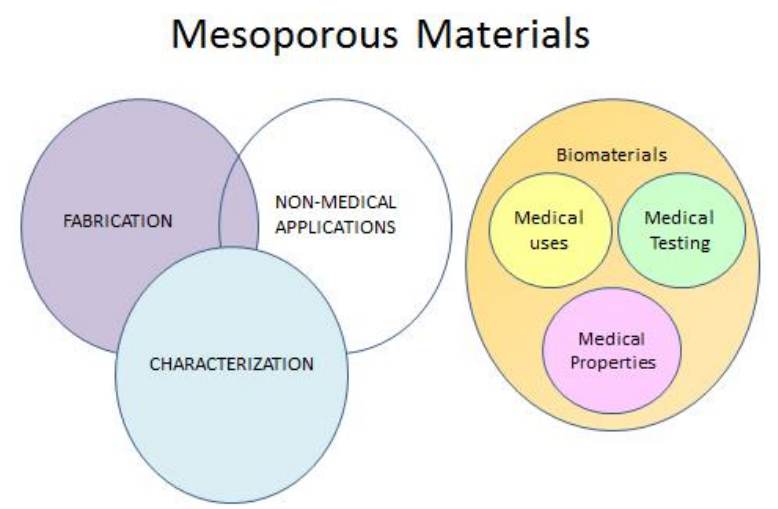

Figure 1: Grouping of retrieved reviews (coloured topics) on mesoporous materials and biomaterials. Reviews which focus on nonmedical and non-biological applications were not included. Reviews which focus on general fabrication techniques and characterization techniques were included.

Nearly 300 reviews have been collated spanning more than 100 different journals. Within each group, reviews are arranged chronologically with the most recent first. The most intensively studied mesoporous biomaterial and medical application area were found to be mesoporous silica and drug delivery respectively, both with more than 50 dedicated reviews over the last decade 2005-2015.

\section{General reviews on mesoporous materials}

1. Recent advances in hybrid periodic mesostructured organosilica materials: opportunities from fundamental to biomedical applications. M.A. Wahab, J.N. Beltramini. RSC Adv. 5. 79129-79151 (2015).

2. Physicochemical properties affect the synthesis, controlled delivery, degradation and pharma- 


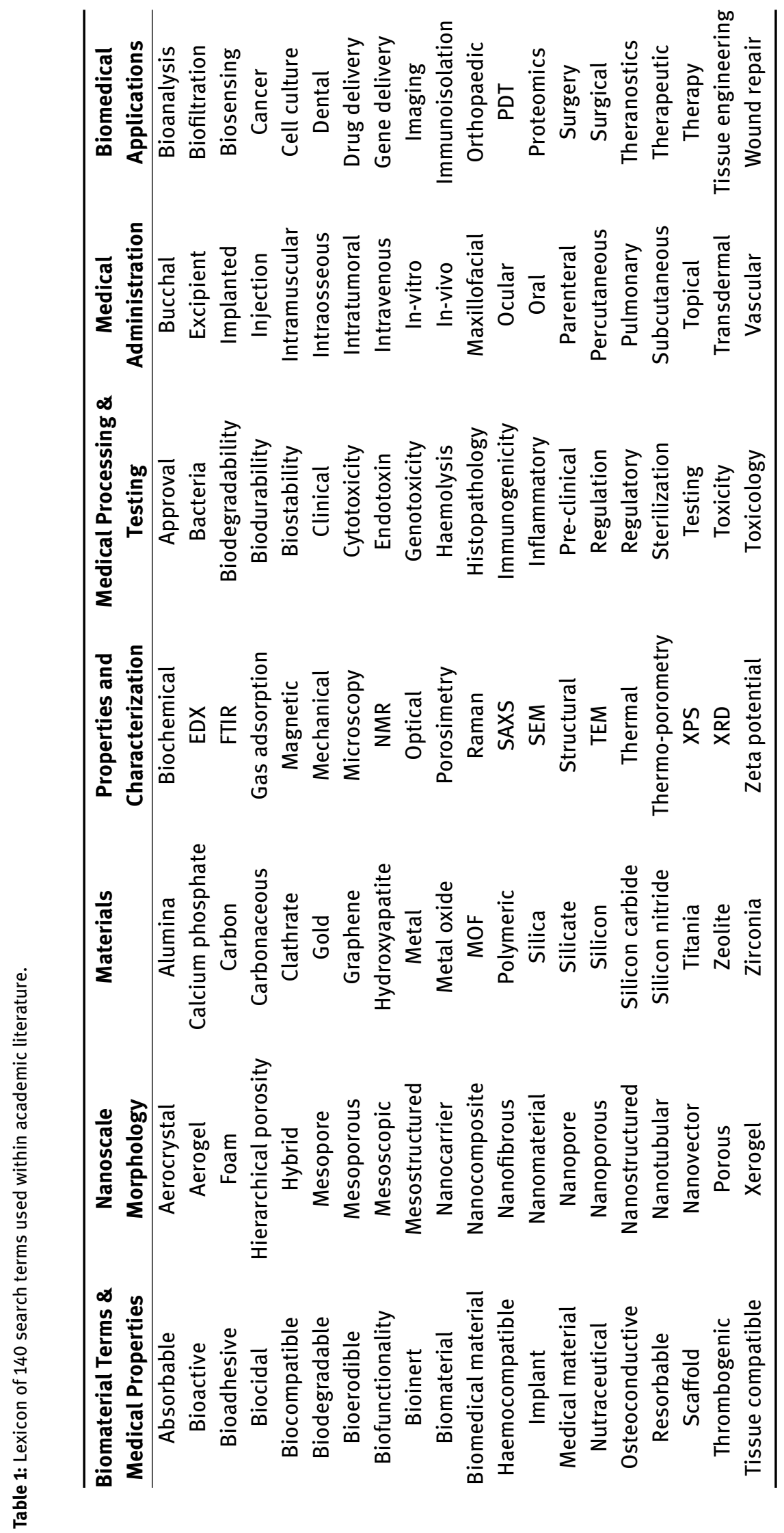


cokinetics of inorganic nanoporous materials. I.K Yazdi, A. Ziemys, M. Evangelopoulos, J.O. Martinez, M. Kojic, E. Tasciotti. Nanomedicine.DOI: 10.2217/nnm.15.133 (2015).

3. Mesoporous materials as multifunctional tools in biosciences : principles and applications. M. Moritz and M. Geske-Moritz. Mater. Sci. Engn.C49, 114-151 (2015).

4. Soft matter in hard confinement: phase transition thermodynamics, diffusion and flow in nanoporous media. P.Huber. J.Phys.Condens.Matter 27, 103102 (43pp) (2015).

5. Silicon: The evolution of its use in biomaterials. J.R. Henstock, L.T.Canham, S.I. Anderson. Acta Biomaterialia 11, 17-26 (2015).

6. Hollow structured mesoporous materials : chemical synthesis, functionalization and applications. Y. Li and J. Shi. Adv. Mater. 26(20) 3176-3205 (2014).

7. Polymeric nanoporous materials fabricated with supercritical $\mathrm{CO}_{2}$ and $\mathrm{CO}_{2}$-expanded liquids. A. Zhang, Q. Zhang, H. Bai, L. Li, J. Li. Chem. Soc. Rev. 43, 6938-6953 (2014).

8. Stimulus-responsive metal organic frameworks. S.S. Nagarkar, A.V. Desai, S.K. Ghosh. Chem. Asian J. 9(9) 2358-2376 (2014)

9. Nanofibrous scaffolds for biomedical applications. K.C.Gupta, A.Haider, Y.Choi, I.Kang. Biomater.Res. 18:5 (2014).

10. Nanoporous metal organic framework materials for smart applications. M.R. Ryder, J.C. Tan. Mater. Sci. Techn. 30(13) 1598-1612 (2014).

11. Recent progress in aerogel science and technology. K. Kanamori. Adv. Porous Mater. 1, 147-163 (2013).

12. Bio-inspired silica-collagen materials: applications and perspectives in the medical field. S.Heinemann, T.Coradin, M.F.Desimone. Biomater.Sci. 1,688-702 (2013).

13. Mesoporous silica nanoparticles in nanotechnology. D.Douroumis, I.Onyesom, M.Maniruzzaman,J.Mitchell. Crit.Rev.Biotechnol. 33(3) 229-245 (2013).

14. Synthesis, properties and applications of porous graphene: a review. P. Russo, A.Hu, G. Compagnini. Nano-Micro. Lett. 5(4) 260-273 (2013).

15. Nanoporous gold electrodes and their applications in analytical chemistry. M.M. Collinson. Analytical Chem. Article 692484, 21 pages (2013).

16. Ordered porous nanomaterials: the merit of small. A.B. Mucia. ISRN Nanotechnology. Article 257047 (2013).
17. Silicon micro- and nanofabrication for medicine. D. Fine, A. Grattoni, R. Goodall, S.S. Bansal, C. Chiappini, S. Hosali, A.L. van de Ven, S. Srinivasan, X. Liu, B. Godin, L. Brousseau 3rd, I.K. Yazdi, J. Fernandez-Moure, E. Tasciotti, H.J. Wu, Y. Hu, S. Klemm, M. Ferrari. Adv Healthcare Mater. 2(5):63266. doi: 10.1002/adhm.201200214. (2013).

18. Nanoarchitectonics for Mesoporous Materials. K. Ariga, A. Vinu, Y. Yamauchi, Q. Ji, J.P. Hill. Bull. Chem. Soc. Jpn. Vol 85, No 1. 1-32 (2012).

19. Multifunctional to multistage delivery systems: The evolution of nanoparticles for biomedical applications. J.O. Martinez, B.S. Brown, N. Quattrocchi, M. Evangelopoulos, M. Ferrari, E. Tasciotti. Chin Sci Bull. 57(31):3961-3971 (2012).

20. Stimuli-responsive polymer brushes for flow control through nanopores. S.P.Adiga, D.W.Brenner. J.Funct.Biomater. 3,239-256 (2012).

21. Structure and functionalization of mesoporous bioceramics for bone tissue engineering and drug delivery. M.Vallet-Regi, I.Izquiredo-Barba, M.Colilla. Philos.Trans.Roy.Soc.A370(1963) 1400-1421 (2012).

22. Magnetic nanocomposites with mesoporous structures: synthesis and applications. J.Liu, S.Z.Qiao, Q.H.Hu, G.Q.Lu. Small 7(4) 425-443 (2011)

23. Metal-Organic Frameworks: A Rapidly Growing Class of Versatile Nanoporous Materials. S.T. Meek, J.A. Greathouse, M.D. Allendorf. Adv. Mater. 23, 249267 (2011).

24. Bioceramics - from bone regeneration to cancer nanomedicine. M.Vallet-Regi, E.Ruiz-Hernandez. Adv.Mater 23 (44) 5177-5218 (2011).

25. Multifunctional hybrids by combining ordered mesoporous materials and macromolecular building blocks. G.J.A.A. Soler-Ilia, O.Azaroni. Chem.Soc.Rev. 40(2) 1107-1150 (2010).

26. Pores in carbon materials - importance of their control. M. Inagaki. New Carbon Materials 24(3) 193-222 (2009).

27. Multifunctional inorganic nanoparticles for imaging, targeting, and drug delivery. M. Liong, J. Lu, M. Kovochich, T. Xia, S.G. Ruehm, A.E. Nel, F. Tamanoi, J.I. Zink. ACS Nano. 2(5):889-96. doi: 10.1021/nn800072t. (2008).

28. New families of mesoporous materials. A. Vinu, T. Mori, K. Ariga. Sci. Technology Advan. Mater. 7753 -771 (2006).

29. Mesopore-modified zeolites: preparation, characterization and applications. Y. Tao, H. Kanoh, L. Abrams, K. Kaneko. Chem. Rev. 106(3) 896-910 (2006). 
30. Chemistry of Aerogels and their applications. A.C. Pierre, G.M. Pajonk. Chem. Rev. 102, 4243-4265 (2002).

31. Non-siliceous mesostructured and mesoporous materials. F. Schuth. Chem. Mater. 13(10) 3184-3195 (2001).

32. Ordered mesoporous materials. U.Ciesla,F.Schuth. Micro.Meso. Mater. 27(2-3) 131-149 (1999).

33. Periodic mesoporous silica-based organic-inorganic nanocomposite materials. A.Sayari,S.Hamoudi. Chem.Mater. 13(10) 3151-3168 (2001).

34. Synthesis of inorganic materials with complex form. S. Mann, G.A. Ozin. Nature 382, 313-318 (1996).

\section{Reviews on fabrication techniques}

1. Molecular and supramolecular switches on mesoporous silica nanoparticles. N. Song, Y-W. Yang. Chem. Soc. Rev. 44, 3474-3504 (2015).

2. Well-ordered nanohybrids and nanoporous materials from gyroid block copolymer templates. H.Y. Hsueh, C.T. Yao, R.M. Ho. Chem. Soc. Rev. DOI 10.1039/C4CS00424H (2015).

3. Nanoporous silica-based protocells at multiple scales for designs of life and nanomedicine. J. Sun, E. Jakobsson, Y. Wang, C.J. Brinker. Life 5, 214-229. Doi: 10.3390/life5010214 (2015).

4. Heterogeneity within order in metal organic frameworks. H. Furukawa, U. Muller, O.M. Yaghi. Angew Chemie Intn. Ed. (2015) in press

5. Host-guest chemistry of mesoporous silica: precise design of location, density and orientation of molecular guests in mesopores. M. Sohmiya, K. Saito, M. Ogawa. Sci. Technol. Adv. Mater. 16. 054201,17pp (2015).

6. Designing advanced functional periodic mesoporous organosilicas for biomedical applications. D. Esquivel P. Van der Voort, F.J. Romero-Salguero. AIMS Mater Sci. Vol 1, Iss 1, 70-86. (2014).

7. Nanoporous hydroxyapatite preparation methods for drug delivery applications. N.F. Mohammad, R. Othman, F. Yee-Yeoh. Rev. Adv. Mater. Sci 38. 138-147 (2014).

8. A perspective on mesoporous $\mathrm{TiO}_{2}$ materials. W. $\mathrm{Li}$, Z. Wu, J. Wang, A.A. Elzatahry, D. Zhao. Chem. Mater. 26(1) 287-298 (2014).

9. Porous anodic aluminium oxide: anodization and template synthesis of functional nanostructures. W. Lee, S.J. Park. Chem. Rev. 114(5) 7487-7556 (2014).
10. Recent advances in the synthesis of hierarchically nanoporous zeolites. K. Na, M. Choi, R. Ryoo. Micro. Meso. Mater 166, 3-19 (2013).

11. Soft templating strategies for the synthesis of mesoporous materials: inorganic, organic-inorganic hybrid and purely organic solids. N. Pal, A. Bhaumik. Adv. Colloid Interface Sci. 189-190, 21-41 (2013).

12. Field-Induced alignment controls of onedimensional mesochannels in mesoporous materials. Y. Yamauchi. J.Ceram. Soc. Japan. 121(9) 831-840 (2013).

13. Synthesis of mesoporous silica nanoparticles. S-H. Wu, C-Y. Mou, H-P. Lin. Chem. Soc. Rev. 42, 3862-3875 (2013).

14. Engineering of Hollow Mesoporous Nanoparticles for Biomedical Applications. Y. Chen, H. Chen, J. Shi. Advanced Porous Materials Vol 1, 34-62 (2013).

15. Formation of mesostructured thin films at the airliquid interface. K.J.Edler, B.Yang. Chem.Soc.Rev. 42, 3765-3776 (2013).

16. Direct synthesis of ordered mesoporous carbons. T. Y. Ma, L. Liu, Z. Y. Yuan. Chem. Soc. Rev. 42, 39774003 (2013).

17. Ecodesign of ordered mesoporous silica materials. C.Gerardin, J.Reboul, M.Bonne, B.Lebeau. Chem.Soc.Rev. 42, 4217-4255 (2013).

18. Recent progress in mesoporous titania materials: adjusting morphology for innovative applications. J.L. Vivero-Escoto, Y. D. Chiang, K. C-W. Wu. Sci. Technol. Adv. Mater.13 1-9 (2012).

19. Design and preparation of porous polymers. D. Wu, F. Xu, B. Sun, R. Fu, H. He, K. Matyjaszewski. Chem. Rev. 112(7) 3959-4015 (2012).

20. Synthetic routes toward MOF nanomorphologies. E.A. Flugel, A. Ranft, F. Haase, B.V. Lotsch. J. Mater. Chem. 22, 10119-10133 (2012).

21. Materiomics: An - omics Approach to Biomaterials Research. S.W. Cranford, J. de Boer, C.A. van Blitterswijk, M.J. Buehler. Adv. Mater. 1-23 (2012).

22. Ordered mesoporous non-oxide materials. Y. Shi, Y. Wan, D. Zhao. Chem. Soc. Rev. 40(7) 3854-3878 (2011)

23. Overview and industrial assessment of synthesis strategies towards zeolites with mesopores. R. Chal, C. Gerardin, M. Bulut, S. van Donk. Chem. Cat. Chem. 3, 67-81 (2011).

24. Biomimetic smart nanopores and nanochannels. X.Hou, W.Guo, L.Jiang. Chem.Soc.Rev. 40, 2385-2401 (2011).

25. Templated nanoscale porous carbons. Y. Xia, Z. Yang, R. Mokaya. Nanoscale 2, Iss 5. 639-659 (2010). 
26. Nanoporous metal foams. B.C. Tappan, S.A. Steiner, E.P. Luther. Angew. Chemie. Int. Ed. 49(27) 45444565 (2010).

27. Synthesis of polymer-mesoporous silica nanocomposites. L. Wei, N. Hu, Y. Zhang. Materials 3,4066-79 (2010)

28. Nanoporous metals by alloy corrosion: formation and mechanical properties. J. Weissmuller, R.C. Newman, H-J Jin, A.M. Hodge, J.W. Kysar. MRS Bulletin Vol 34, 577-586 (2009).

29. Tuneable porous carbonaceous materials from renewable sources. R.J.White, V.Budarin, R. Luque, J.H. Clark and D.J. Macquarrie. Chem. Soc. Rev. 38, 3401-3418 (2009).

30. Mesoporous materials: tunable structure, morphology and composition. Z.Yang, Y.Lu, Z.Yang. Chem.Commun. 2270-2277 (2009).

31. Advances in microwave assisted synthesis of ordered mesoporous materials. Y. Cao , H.J. Wei, Z. Xia. Trans. Non Ferrous Met. Soc. China 19, s656-664 (2009)

32. Nanoporous colloids: building blocks for a new generation of structured materials. Y. Wang, A.D. Price, F. Caruso. J. Mater. Chem. 19. 6451-6464 (2009).

33. Nanoporous plasmonic metamaterials. J. Biener, G.W. Nyce, A.M. Hodge, M.M. Biener, A.V. Hamza, S.A. Maier. Adv. Mater. 120 (6) 1211-1217 (2008).

34. Microwave synthesis of zeolite membranes: a review. Y. Li, W. Yang. J. Membrane Science 316. 3-17 (2008).

35. Templating mesoporous zeolites. K. Egeblad, C.H. Christensen, M. Kustova, C.H. Christensen. Chem. Mater. 20(3) 946-960 (2008).

36. Templating nanoporous polymers with ordered block copolymers. D.A. Olsen, L. Chen, M.A. Hillmyer. Chem. Mater. 20, 869-890 (2008).

37. Supramolecular aggregates as templates: ordered mesoporous polymers and carbon. Y. wen, Y. Shi, D. Zhao. Chem. Mater. 20(3) 932-945 (2008)

38. Mesoporous carbon materials: synthesis and modification. C. Liang and Z. Li. Angewandte Chemie 47(20) 3696-3717 (2008).

39. Silica-based mesoporous organic-inorganic hybrid materials. F. Hoffmann, M. Cornelius, J. Morell, M. Fröba. Angew. Chem. Ind. Ed. 45. 3216-3251 (2006).

40. Recent progress in the synthesis of porous carbon materials. J.Lee, J.Kim, T.Hyeon. Adv.Mater. 18(16) 2073-2094 (2006).

41. Bioinspired synthesis of mesoporous silicas. Q. Sun, E.G. Vrieling, R.A. van Santen, N.A.J.M. Sommerdijk. Curr. Opin. Solid State. Mater Sci. 8, 111-120 (2004).
42. Synthesis of new nanoporous carbon materials using nanostructured silica materials as templates. J. Lee, S. Han, T. Hyeon. J. Mater. Chem. 14, 478-486 (2004).

43. Chemical strategies to design textured materials: from microporous and mesoporous oxides to nanonetworks and hierarchical structures. G.J. de.A.A. Soler-lllia, C. Sanchez, B. Lebeau, J. Patarin. Chem. Rev. 102, 4093-4138 (2002).

\section{Reviews on characterisation techniques}

1. Transport phenomena in nanoporous materials. J. Karger. Chem Phys Chem 16(1) 24-51 (2015).

2. Mesoporous bioactive glasses: Relevance of their porous structure compared to that of classical bioglasses. I. Izquierdo-Barba, M. Vallet-Regi. Biomed. Glasses 1, 140-150 (2015).

3. Structures of Silica-Based Nanoporous Materials Revealed by Microscopy. L. Han, T. Ohsuna, Z. Liu, V. Alfredsson, T. Kjellman, S. Asahina, M. Suga, Y. Ma, P. Oleynikov, K. Miyasaka, A. Mayoral, I. Diaz, Y. Sakamoto, S.M. Stevens, M.W. Anderson, C. Xiao, N. Fujita, A. Garcia-Bennett, K. B. Yoon, S. Che, O. Terasaki. Zeitsch. Anorg. Allgem. Chemie. Vol 640. Iss 3-4. 521-536. (2014).

4. Recent advances in solid state NMR of small molecules in confinement. M. Werner, N. Rothermel, H. Breitzke, T. Gutmann, G. Buntkowsky. Isr. J. Chem. 54(1-2) 60-73 (2014).

5. Physical adsorption characterization of nanoporous materials: progress and challenges. M.Thommes, K.A.Cychosz. Adsorption 20: 233-250 (2014).

6. Characterization of protein unfolding with solidstate nanopores. J.Li, D.Fologea, R.Rollings, B.Ledden. Protein Pept.Lett. 21(3) 256-265 (2014).

7. A review of fine structures of nanoporous materials as evidenced by microscopic methods. Z. Liu, N. Fujita, K. Miyasaka, L. Han, S.M. Stevens, M. Suga, S. Asahina, B. Slater, C. Xiao, Y. Sakamoto, M.W. Anderson, E. Ryoo, O. Terasaki. Microscopy 62 (1) 109-146 (2013)

8. Diffraction analysis of mesostructured mesoporous materials. L.A.Solovyov. Chem.Soc.Rev. 42, 37083720 (2013).

9. Density functional theory methods for characterization of porous materials. J.Landers, G.Y.Gor, A.V.Neimark. Coll.Surf.A437, 3-32 (2013).

10. The complexity of mesoporous silica nanomaterials unravelled by single molecule mi- 
croscopy. T.Lebold,J.Michaelis, C.Brauchle. Phys.Chem.Chem.Phys. 13, 5017-5033 (2011).

11. Utilising thermoporometry to obtain new insights into nanostructured materials- review part 2 . J.Riikonen, J.Salonen, V.Lehto. J. Therm. Anal. Calorim. 105(3) 823-830 (2011).

12. Utilising thermoporometry to obtain new insights into nanostructured materials- review part 1. J.Riikonen, J.Salonen, V.Lehto. J. Therm. Anal. Calorim. : 105(3) 811-821 (2011).

13. Reporters in the nanoworld: diffusion of single molecules in mesoporous materials. J.Michaelis, C.Brauchle. Chem.Soc.Rev. 39, $4731-4740$ (2010).

14. Physical adsorption characterization of nanoporous materials. M.Thommes. Chemie Ingenieur Technik 82(7) 1059-1073 (2010).

15. Mercury Porosimetry : a General (Practical) Overview. H.Giesche. Part. Part. Syst. Charact.23, 1-11 (2006).

16. Small Angle X-Ray Scattering to Characterize Nanostructures in Inorganic and Hybrid Materials Chemistry. H. Peterlik and P. Fratzl. Monatshefte Fur Chemie 137, 529-543 (2006).

17. Positron annihilation as a method to characterize porous materials. D.W.Gidley, H.G.Peng, R.S.Vallery. Annu.Rev.Mater.Res. 36: 49-79 (2006).

18. Physisorption hysteresis loops and the characterization of nanoporous materials. K.S.W.Sing, R.T.Williams. Ads.Sci.Techn. 22(10) 773-782 (2004).

19. Pore size determination in modified micro- and mesoporous materials. Pitfalls and limitations in gas adsorption analysis. J.C. Groen, L.A.A. Peffer, J. Perez-Ramirez. Micro. Meso. Mater. 60, 1-17 (2003).

20. Three-dimensional electron microscopy of mesoporous materials-recent strides towards spatial imaging at the nanometer scale. K.P. de Jong, A.J. Koster. Chem. Phys. Chem. 3, 776-780 (2002).

21. Gas adsorption characterization of ordered organicinorganic nanocomposite materials. M. Kruk, M. Jaroniec. Chem. Mater.13(10) 3169-3183 (2001)

22. The structural and luminescence properties of porous silicon. A.G. Cullis, L.T. Canham, P.D.J. Calcott. J. Appl. Phys. 82 (3), (1997).

\section{Reviews on toxicity and biocompatibility}

1. Biocompatibility of mesoporous silica nanoparticles? Y.Shi,M.L.Miller,A.J.DiPasqua. Comments Inorg.Chem. DOI: 10.1080/02603594.2015.1088439 (2015).
2. Shape-mediated biological effects of mesoporous silica nanoparticles. N. Hao, L. Li, F. Tang. J.Biomed. Nanotechn. 10, 2508-2538 (2014)

3. Zeolite and mesoporous silica nanomaterials: greener synthesis, environmental applications and biological toxicity. S.E. Lehman and S.C. Larsen. J. Environ. Sci. Nano. 1, 200-213 (2014).

4. In vivo bio-safety evaluations and diagnostic/therapeutic applications of chemically designed mesoporous silica nanoparticles. Y. Chen, H. Chen and J. Shi. Adv. Mater. 25(23) 3144-3176 (2013).

5. A concise review of carbon nanotube's toxicity. S.Y. Madani, A. Mandel, A.M. Seifalian. Nano Reviews 4:21521 (2013).

6. Mesoporous silica nanoparticle nanocarriers biofunctionality and biocompatibility. D.Tarn, C.E.Ashley, M.Xue,E.C.Carnes, J.I.Zink, C.J.Brinker. Acc.Chem.Res. 46(3) 792-801 (2013).

7. Critical Considerations in the Biomedical Use of Mesoporous Silica nanoparticles. Y-S. Lin, K.R. Hurley, C.L. Haynes. J. Phys. Chem. Lett.3 (3) 364-374 (2012).

8. The toxicological mode of action and the safety of synthetic amorphous silica - a nanostructured material. C. Frujitier-Polloth. Toxicology. 294 (2-3) 61-79 (2012).

9. Biocompatibility of Mesoporous Silica Nanoparticles. T. Asefa, Z. Tao. Chem Res. Toxicol 25 (11) 22652284 (2012).

10. Biocompatibility assessment of Si-based nano- and micro- particles. H. Jaganathan, B. Godin. Adv. Drug. Deliv Rev. 64(15) 1800-1819 (2012).

11. The nanosilica hazard: another variable entity. D. Napierska, L.C.J. Thomassen, D. Lison, J.A .Martens, P.H. Hoet. Particle \& Fibre Toxicology. 7:39 (2010).

12. Improving biocompatibility of implantable metals by Nanoscale modification of surfaces: an overview of strategies, fabrication methods and challenges. F. Variola, F. Vetrone, L. Richert, P. Jedrzejowski, J-H. Yi, S. Zalzal, S. Clair, A. Sarkissian, D.F. Perepichka, J.D. Wuest, F. Rosei, A. Nanci. Small 5(9) 996-1006 (2009).

13. A review of carbon nanotube toxicity and assessment of potential occupational and environmental health risks. C.W. Lam, J.T. James, R. McCluskey, S. Arepalli, R.L. Hunter. Crit. Rev. Toxicol. 36(3) 189-217 (2006). 


\section{Reviews on mesoporous silica, organosilicas and silicates}

1. Mesoporous silica nanoparticles in drug delivery and biomedical applications. Y. Wang, Q. Zhao, N. Han, L. Bai, J. Li, J. Liu, E. Che, L. Hu, Q. Zhang, T. Jiang, S. Wang. Nanomedicine 11(2). 313-327 (2015).

2. Silica-based mesoporous nanobiomaterials as promoter of bone regeneration process. N. Shadjou, M. Hasanzadeh. J. Biomed. Mater. Res. A. DOI:10.10032/jbm.a.35504.(2015).

3. Mesoporous silica-based nanodevices for biological applications. Z. Tao. RSC Adv. 4, 18961-18980 (2014)

4. Drug delivery/imaging multifunctionality of mesoporous silica-based composite nanostructures. Y. Chen, H. Chen, J. Shi. Expert Opin. Drug Deliv. 11(6) 917-30 (2014).

5. Periodic mesoporous organosilicas for advanced applications. S.S. Park, M.S. Moorthy, C.S. Ha. NPG Asia Materials 6, doi 10.1038/am.2014.13, 21pages (2014).

6. Mesoporous silica nanoparticles in medicine-recent advances. V. Mamaeva, C. Sahlgen, M. Linden. Adv. Drug Del Rev. 65 (5) 689-702 (2013).

7. Applications of mesoporous silica in food - a review. A. Bernardos and L. Kourimska. Czech J. Food Sci. 31(2) 99-107 (2013).

8. Mesoporous silica films as a novel biomaterial: applications in the middle ear. N.Ehlert, P.P.Mueller, M.Stieve, T.Lenarz, P.Behrens. Chem.Soc.Rev. 42(9) 3847-3861 (2013).

9. Mesoporous silica nanoparticles: a multifunctional nano therapeutic system. W.X.Mai, H.Meng. Integr.Biol. 5,19-28 (2013).

10. A Review: Fundamental Aspects of Silicate Mesoporous Materials. Z.A. ALOthman. Materials. 5. 28742902 (2012).

11. Mesoporous bioactive glasses: structure characteristics, drug/growth factor delivery and bone regeneration application. C. Wu, J. Chiang. Interface Focus 2, 292-306 (2012).

12. Mesoporous silica nanoparticles: their projection in nanomedicine. M. Vallet-Regi. ISRN Mat. Sci. doi 10.5402/2012/608548, 20 pages (2012).

13. Mesoporous silica nanoparticles: synthesis, biocompatibility and drug delivery. F. Tang, L. Li, D. Chen. Adv. Mater. 24(12) 1504-1534 (2012).

14. Mesoporous silica nanoparticles in biomedical applications. Z. Li, C.J. Barnes, A. Bosoy, J.F. Stoddart, J.I. Zink. Chem. Soc. Rev. 41(7). 2590-605. Doi: 10.1039/c1cs15246g. (2012).
15. Synthesis, toxicology and potential of ordered mesoporous materials in nanomedicine. A.E. GarciaBennett. Nanomedicine (Lond). 6 (5), 867-77 (2011).

16. Mesoporous silica nanoparticles for bioadsorption, enzyme immobilisation and delivery carriers. A. Popat, S.B. Hartono, F. Stahr, J. Liu, S.Z. Qiao, M.L.G. Qing. Nanoscale 3(7) 2801-2818 (2011).

17. Mesoporous silica nanoparticle based nano drug delivery systems: synthesis, controlled drug release and delivery, pharmacokinetics and biocompatibility. Q.He, J.Shi. J.Mater.Chem. 21, 5845-5855 (2011).

18. Mesoporous silica nanoparticles as nanocarriers. S.H.Wu, Y.Hung, C.Y.Mou. Chem.Commun.47,99729985 (2011).

19. Nanostructured mesoporous silica matrices in nanomedicine. M. Vallet-Regi. J. Internal Medicine 267(1) 22-43 (2010).

20. Recent development, applications, and perspectives of mesoporous silica particles in medicine and biotechnology. L. Pasqua. S. Cundari, C. Ceresa, G. Cavaletti. Curr. Med. Chem. 16(23). 3054-63 (2009).

21. Mesoporous silica nanomaterial-based biotechnological and biomedical delivery systems. S. Giri, B.G. Trewyn, V.S. Lin. Nanomedicine (Lond). 2(1). 99-111 (2007).

22. Mesoporous silica nanoparticles for drug delivery and biosensing applications. I.S. Slowing, B.G. Trewyn, S. Giri, V.S.Y. Lin. Adv. Funct. Mater. 17, 12251236 (2007).

23. Past, present and future of periodic mesoporous organosilicas - the PMOs. B. Hatton, K. Landskron, W. Whitnall, D. Perovic, G.A. Ozin. Acc. Chem. Res. 38(4) 305-312 (2005).

24. Hybrid inorganic-organic mesoporous silicatesnanoscopic reactors coming of age. A. Stein, B.J. Melde, R.C. Schroden. Adv. Mater. 12(19) 1403-1419 (2000).

\section{Reviews on mesoporous silicon}

1. Nanostructured porous silicon: the winding road from photonics to cell scaffolds - a review. J. Hernandez-Montelongo, A. Munoz-Noval, J. Predestinacion Garcia-Ruiz, V. Torres-Costa, R.J. MartinPalma, M. Manso-Silvan. Frontiers in Bioeng. Biotech. Vol 3, Article 60 , p1-15 (2015).

2. Porous silicon nanoparticles for nanomedicine: preparation and biomedical applications. H.A. Santos, E. Mäkilä, A.J. Airaksinen, L.M. Bimbo, and J. Hirvonen. Nanomedicine 9(4) 535-554 (2014). 
3. Porous silicon advances in drug delivery and immunotherapy. D.J. Savage, X. Lin, S.A. Curley, M. Ferrari, R.E. Serda. Curr. Opin. Pharmacol. 13(5) 834-841 (2013).

4. Porous silicon and its application in biology and medicine. O.I. Ksenofontanova, A.V. Vasin, V.V. Egorov, A.V. Bobyl, F Y. Soldatenkov, E.I. Terukov, V.P. Ulin, N.V. Ulin, and O.I. Kiselev. Technical Physics Vol 59 Iss. 1. 66-77 (2013).

5. Functionalised porous silicon as a biosensor: emphasis on monitoring cells in vivo and in-vitro. B. Gupta, Y. Zhu, B. Guan, P.J. Reece, J.J. Gooding. Analyst 138(13) 3593-3615 (2013)

6. Porous silicon biosensor: current status. S. Dhanekar, S. Jain. Biosens. Bioelectr. 41,54-64 (2013)

7. The photophysics of porous silicon: technological and biomedical applications. G.E. Kotkovskiy, Y.A. Kuzishchin, I. L. Martynov, A.A. Chistyakov and I. Nabiev. Phys. Chem. Chem. Phys. (2012).

8. Multi-stage deliver nano-particle systems for therapeutic applications. R.E.Serda, B.Godin, E. Blanco, C. Chiappini, M. Ferrari. Biochim. Biophys. Acta 1810(3) 317-329 (2011).

9. Nanostructured porous silicon- polymer hybrids: from biosensing to drug delivery. L.M.Bonano, E.Segal. Nanomedicine 6(10) 1755-1770 (2011).

10. Multifunctional porous silicon for therapeutic drug delivery and imaging. H.A. Santos, L.M. Bimbo, V.P. Lehto, A.J. Airaksinen, J. Salonen, J. Hirvonen. Curr Drug Discov Technol. 8(3):228-49. (2011).

11. Biomedical applications of nanostructured porous silicon: a review. R.J. Martín-Palma, M. MansoSilván, V. Torres-Costa. J. Nanophotonics, Vol 4. 042502 (2010).

12. Porous silicon - a versatile host material. P. Granitzer, K. Rumpf. Materials 3, 943-998 (2010).

13. The importance of surface chemistry in mesoporous materials: lessons from porous silicon biosensors. K.A. Kilian, T. Böcking, J.J. Gooding. Chem. Commun. 630-640 (2009).

14. Porous silicon biosensors on the advance. A. Jane, R. Dronov, A.Hodges, N.H. Voelcker. Trends Biotechnol. 27(4) 230-239 (2009)

15. Fabrication and chemical surface modification of porous silicon for biomedical applications. J.Salonen, V.P. Lehto. Chem. Engn. J. 137(1) 162-172 (2008).

\section{Reviews on mesoporous carbon and carbonaceous materials}

1. Mesoporous carbon biomaterials. Y. Chen, J. Shi. Sci. China. Mater. 58. 241-257 (2015).

2. Three dimensional macroporous architectures and aerogels built of carbon nanotubes and/or graphene: synthesis and applications. S. Nardecchia, D. Carriazo, M.L. Ferrer, M.C. Gutierrez, F. del Monte. Chem. Soc. Rev. 42, 794-830 (2013).

3. Carbon-based nanomaterials: multi-functional materials for biomedical engineering. C. Cha, S.R. Shin, N. Annabi, M.R. Dokmeci, A. Khademhosseini. ACS Nano 7(4) 2891-2897 (2013).

4. Nanoporous membranes derived from block copolymers: from drug delivery to water purification. E.A.Jackson,M.A.Hillmyer. ACS Nano. 4(7) 3548-3553 (2010).

5. Carbon nanofibers and carbon nanotubes in regenerative medicine. P.A. Tran, L. Zhang, T.J. Webster. Adv. Drug Deliv. Rev. 61, 1097-1114 (2009).

6. Mechanical and biological properties of nanoporous carbon membranes. R.J. Narayan. R. Aggarwal, W. Wei, C. Jin, N.A. Monteiro-Riviere, R. Crombez, W. Shen. Biomedical Materials Vol 3 No 3, 034107, doi: 1088/1748-6041 (2008).

7. Applications of carbon nanotubes-based biomaterials in biomedical nanotechnology. S. Polizu, $\mathrm{O}$. Savadogo, P. Poulin, L'H. Yahia. J. Nanosci. Nanotech. Vol 6, 1883-1904 (2006).

\section{Reviews on mesoporous calcium phosphates}

1. Nanoporous Hydroxyapatite Preparation Methods for Drug Delivery Applications. N.F. Mohammad, R. Othman, F. Yee-Yeoh. Rev. Adv. Mater. Sci. 38. 138-147 (2014).

2. Calcium Orthophosphate-Based Bioceramics. S.V. Dorozhkin. Materials 6. 3840-3942. (2013).

3. Nanostructured calcium phosphates: preparation and their applications in biomedicine. F. Chen, Y. $\mathrm{Zhu}$, J. Wu, P. Huang, D. Cui. Nano. Biomed. Eng. 4(1) 41-49 (2012).

4. Synthesis and application of nanostructured calcium phosphate ceramics for bone regeneration. D.A. Cardoso, J.A. Jansen, S.C.G. Leeuwenburgh. J. Biomed. Mater. 100B (8) 2316-2326 (2012).

5. Calcium phosphate ceramic systems in growth factor and drug delivery for bone tissue engineering: a 
review. S. Bose, S. Tarafder. Acta Biomater. 8(4) 14011421 (2012).

6. Calcium phosphate cements as bone drug delivery systems: A review. M.P. Ginebra, T. Traykova, J.A. Planell. J. Controlled Rel. 113. 102-110 (2006).

\section{Reviews on mesoporous metals, metal oxides and metal organic frameworks}

1. Mesoporous alumina as a biomaterial for biomedical applications. E. Xifre-Perez, J. Ferre-Borull, J. Pallares, L.F. Marsal. Mesoporous Biomater 2:13-32 (2015).

2. Synthesis of non-siliceous mesoporous oxides. D. Gu, F. Schüth. Chem. Soc. Rev. 43. 313-344 (2014).

3. A Review on Bioactive Porous Metallic Biomaterials. K. Mediaswanti, C. Wen, E.P. Ivanova, C.C. Berndt, F. Malherbe, V.T.H. Pham, and J. Wang. J. Biomim Biomater Tissue Eng Vol 18. Iss 1. (2013).

4. Where bio meets nano: The many uses for nanoporous aluminum oxide in biotechnology. C.J. Ingham, J. ter Maat, W.M. de Vos, Biotechnology Advances 30, 1089-1099 (2012).

5. Ordered mesoporous metal oxides: synthesis and applications. Y. Ren, Z. Ma, P.G. Bruce. Chem. Soc. Rev. 41, 4909-4927 (2012).

6. Mesoporous metal-organic framework materials. W.Xuan, C.Zhu, Y.Liu, Y.Cui. Chem.Soc.Rev. 41,16771695 (2012).

7. Recent progress in mesoporous titania materials: adjusting morphology for innovative applications. J.L. Vivero-Escoto, Y-D. Chiang, K.C-W. Wu, Y. Yamauchi. Sci. Technol. Adv. Mater. 13013003 (2012).

8. Nanoscale surface modifications of medically relevant metals: state-of-the art and perspectives. F. Variola, J.B. Brunski, G. Orsini, P. Tambasco de Oliveire, R. Wazen, A. Nanci. Nanoscale 3, 335-353. (2011).

9. Nanoscale metal-organic framworks for biomedical imaging and drug delivery. J. Della Rocca, D. Liu, D. Lin. Acc. Chem. Res. 44(10) 957-968 (2011).

10. Biomedical applications of metal organic frameworks. S.Keskin, S.Kizilel. Ind. Eng. Chem. Res. 50(4) 1799-1812 (2011).

11. BioMOFs: metal organic frameworks for biological and medical applications. A.C. McKinlay, R.E. Morris, P. Horcajada, G. Férey, R. Gref, P. Couvreur, C. Serre. Angew. Chem. Int. Ed. Engl. 23(36) 6260-6266 (2010).

\section{Reviews on drug delivery}

1. Advances in mesoporous silica nanoparticles for targeted stimuli-responsive drug delivery. A Baeza, $\mathrm{M}$ Colilla, M.Vallet-Regi. Expert Opinion on Drug Delivery. Vol 12, No 2. 319-337 (2015).

2. Large pore mesoporous silica nanomaterials for application in delivery of biomolecules. N.Z. Knezevic, J-O. Durand. Nanoscale 14; 7(6). $2199-2209$ (2015).

3. Novel Delivery Systems for Improving the Clinical Use of Peptides. M. Kovalainen, J. Mönkäre, J. Riikonen, U. Pesonen, M. Vlasova, J. Salonen, V-P. Lehto, K. Jarvinen, K-H. Herzig. Pharmacol. Rev. 67. 541-561 (2015).

4. Mesoporous silica nanoparticles in target drug delivery system: A review. C. Bharti, U. Nagaich, A.K. Pal. N. Gulati. Int. J. Pharm. Invest. Vol 5, Issue 3. 124-133, (2015).

5. Mesoporous silica formulation strategies for drug dissolution enhancement: a review. C.A McCarthy, R.J. Ahern, R. Dontireddy, K.B. Ryan, A.M. Crean. Expert Opin. Drug Deliv. 7, 1-16 (2015).

6. Therapeutic Potential of Inorganic Nanoparticles for the Delivery of Monoclonal Antibodies. C.T. Turner, S.J.P. McInnes, N.H. Voelcker, A.J. Cowin. J. Nanomater. Article ID 309602, 11 pages, (2015).

7. Gated supramolecular chemistry in hybrid mesoporous silica nanoarchitectures: controlled delivery and molecular transport in response to chemical, physical and biological stimuli. S.Alberti, G.J.A.A.Soler-Illia, O.Azzaroni. Chem.Commun. In press (2015).

8. Large pore mesoporous silica nanomaterials for application in delivery of biomolecules. N.Z. Knežević, J-O. Durand. Nanoscale 7, 2199-2209 (2015).

9. Multifunctional mesoporous bioactive glasses for effective delivery of therapeutic ions and drug/growth factors. C.Wu, J.Chang. J.Contr.Release 193, 282-295 (2014).

10. Multifunctional mesoporous silica nanoparticles as a universal platform for drug delivery. C. Argyo, V. Weiss, C. Brauchle, T. Bein. Chem. Mater. 26(1) 435451 (2014).

11. An emerging platform for drug delivery: Aerogel based systems. Z. Ulker, C. Erkey. J. Control. Rel. 177, 51-63. (2014).

12. Packaging biological cargoes in mesoporous materials: opportunities for drug delivery. J. Siefker, P. Karande and M.O. Coppens. Expert Opin. Drug Deliv. 11(11) 1781-1793 (2014). 
13. Nanostructured porous silicon-mediated drug delivery. R.J. Martín-Palma, J. Hernández-Montelongo, V. Torres-Costa, M. Manso-Silván and A. Munoz-Noval. Informa healthcare.com (2014).

14. The practicality of mesoporous silica nanoparticles as drug delivery devices and progress toward this goal. R. Roggers, S. Kanvinde, S. Boonsith, D. Oupický. AAPS Pharm. Sci Tech. 1530-9932 (2014).

15. Mesoporous silica nanoparticles: importance of surface modifications and its role in drug delivery. S.K.Natarajan, S.Selvaraj. RSC Adv. 4, 14328-14334 (2014).

16. Mesoporous silica nanoparticles: a potential targeted delivery vector for reproductive biology? N. Barkalina, C. Jones, K. Coward. Nanomedicine. 9(5) 557-560 (2014).

17. Applications of mesoporous materials as excipients for innovative drug delivery and formulation. S.C. Shen, W.K. Ng, L.S. Chia, Y.C. Dong, R.B. Tan. Curr. Pharm. Des. 19(35) 6270-6289 (2013).

18. Porous silicon advances in drug delivery and immunotherapy. D.J. Savage, X, Lin, S.A. Curley, M. Ferrari, and R.E. Serda. Curr Opin Pharmacol. 13 (5). 834-41. (2013).

19. Mesoporous silica nanoparticles as antigen carriers and adjuvants for vaccine delivery. K.T. Mody, A. Popat, D. Mahony, A.S. Cavallaro, C. Yu, N. Mitter. Nanoscale DOI: 10.1039/c3nr00357d. (2013).

20. Metal organic frameworks as potential drug delivery systems. C.Y. Sun, X.L Wang. Z.M. Su. Expert Opin. Drug Deliv. 10(1) 89-101 (2013).

21. Silica-based mesoporous nanoparticles for controlled drug delivery. S. Kwon, R.K. Singh, R.A. Perez, E.A. Abou Neel, H-W Kim, and W. Chrzanowski. J. of Tissue Engineering. 4. 1-18 (2013).

22. Recent advances in porous silicon technology for drug delivery. T.J. Barnes, K.L. Jarvis, C.A. Prestidge. Therapeutic Delivery Vol 4, No 7. 811-823 (2013).

23. Mesoporous materials and nanocrystals for enhancing the dissolution behavior of poorly water-soluble drugs. H.A. Santos, L. Peltonen, T. Limnell, J. Hirvonen. Curr Pharm Biotechnol. 14(10):926-38 (2013).

24. Functional nanoporous membranes for drug delivery. G. Jeon, S.Y. Yang, J.K. Kim. J. Mater. Chem. Vol 22. Iss 30. 14814-14834 (2012).

25. Nanostructured porous Si-based nanoparticles for targeted drug delivery. M-A. Shahbazi, B. Herranz, H.A. Santos. Biomatter 2: 4. 296-312. (2012).

26. Functionalized mesoporous silica materials for controlled drug delivery. P. Yang, S. Gai, J. Lin. Chem. Soc. Rev. 14, Iss 9, 3679-3698 (2012).
27. Application of mesoporous silicon dioxide and silicate in oral amorphous drug delivery systems. K.K. Qian, R.H. Bogner. J. Pharm. Sci. 101(2) 444-463 (2012).

28. Surface chemistry of porous silicon and implications for drug encapsulation and delivery applications. K.L. Jarvis, T.J. Barnes, C.A. Prestidge. Adv Colloid Interface Sci. 175, 25-38. doi: 10.1016/j.cis.2012.03.006.(2012).

29. Silica materials in drug delivery applications. S. Simovic, N. Ghouchi-Eskandar, A.M. Sinn, D. Losic, C.A. Prestidge. Curr Drug Discov Technol. 8(3):26976. (2011).

30. Drug delivery from structured porous inorganic materials. M. Arruebo. Advanced Review, Nanomedicine and Nanobiotechnology Vol 4, Iss 1. 16-30. (2011).

31. Polysaccharide-based aerogels- promising biodegradable carriers for drug delivery systems. C.A. Garcia-Gonzalez, M.Alnaief and I. Smirnova. Carbohydrate Polymers 86, 1425-1438 (2011).

32. Mesoporous Materials as Controlled Drug Delivery Formulations. H.A. Santos, J. Salonen, L.M. Bimbo, V-P. Lehto, L. Peltonen, J. Hirvonen. J. Drug Deliv. Sci. Tech. 21(2), 139-155. (2011).

33. Nanoporous inorganic membranes or coating for sustained drug delivery in implantable devices. E. Gultepe, D. Nagesha, S. Sridhar, M. Amiji. Advan. Drug Del. Rev. 62. 305-315 (2010).

34. Mesoporous silica nanoparticles for intracellular controlled drug delivery. J.L.Vivero-Escoto, II Slowing, B.G.Trewyn, V.S.Lin. Small 6(18) 1952-1967 (2010).

35. Metal-Organic Frameworks as Potential Drug Carriers. R. C. Huxford, J.Della Rocca, W. Lin. Curr. Opin. Chem. Biol. 14(2) 262-268 (2010).

36. Towards multifunctional, targeted drug delivery systems using mesoporous silica nanoparticlesopportunities \& challenges. J.M. Rosenholm, C. Sahlgren, M. Lindén. Nanoscale. 2(10):1870-83. doi: 10.1039/c0nr00156b. (2010).

37. Capped mesoporous silica nanoparticles as stimuliresponsive controlled release systems for intracellular drug/gene delivery. Y. Zhao, J.L. Vivero-Escoto, I.I. Slowing. Expert Opin. Drug Deliv. 7(9) 1013-1029 (2010).

38. Inorganic nanoporous membranes for immunoisolated cell-based drug delivery. A. Mendelsohn, T. Desai. Adv. Exp Med Biol. 670. 104-125. (2010). 
39. Ordered mesoporous silicates as matrices for controlled release of drugs. T. Ukmar, O. Planinsek. Acta. Pharm. 60, 373-385 (2010).

40. Ordered mesoporous materials for drug delivery. S.Wang. Micro.Meso.Mater. 117(1-2) 1-9 (2009).

41. Self-ordered nanopore and nanotube platforms for drug delivery applications. D. Losic, S. Simovic. Iforma UK. Expert Opinion on Drug Deliv. Vol 6, No 12. 1363-1381 (2009).

42. Silica xerogels as pharmaceutical drug carriers. D. Quintanar-Guerrero, A. Ganem-Quintanar, M.G. Nava-Arzaluz, E. Pinon-Segundo. Expert Opin Drug Deliv. 6 (5). 485-98 (2009).

43. Drug delivery from ordered mesoporous matrices. M. Manzano, M. Colilla, M. Vallet-Regi. Expert Opin. Drug Deliv. 6(12) 1383-1400 (2009).

44. Silicon-polymer hybrid materials for drug delivery. S.J.P.McInnes, N.H.Voelcker. Future Med. Chem. 1(6) 1051-1074 (2009).

45. Mechanised nanoparticles for drug delivery. K.K. Cotí, M.E. Belowich, M. Liong, M.W. Ambrogio, Y.A. Lau, H.A. Khatib, J.I Zink, N.M. Khashab, J.F. Stoddart. Nanoscale. 1(1):16-39. doi: 10.1039/b9nr00162j. Epub (2009).

46. Porous silicon in drug delivery devices and materials. E.J. Anglin, L. Cheng, W.R. Freeman, M.J. Sailor. Adv. Drug Deliv. Rev 60 (11). 1266-1277 (2008).

47. Mesoporous silica nanoparticles as controlled release drug delivery and gene transfection carriers. I.I. Slowing, J.L. Vivero-Escoto, C.W. Wu, V.S.Y. Lin. Adv. Drug Deliv. Reviews 60, 1278-1288 (2008).

48. Engineering silica particles as oral drug delivery vehicles. S.P.Rigby, M.Fairhead, C.F.van der Walle. Curr.Pharm.Des. 14(18) 1821-1831 (2008).

49. Mesoporous silicon in drug delivery applications. J. Salonen, A.M. Kaukonen, J. Hirvonen, V.P. Lehto. J. Pharm. Sci. 97(2) 632-53 (2008).

50. Mesoporous materials for drug delivery. M. ValletRegi, F. Balas, D. Arcos. Angew. Chemie. 46(40) 7548-7558 (2007)

51. Mesoporous silicon : a platform for the delivery of therapeutics. C.A. Prestidge, T.J. Barnes, C-H Lau, C. Barnett, A. Loni, L.T. Canham. Expert. Opin. Drug Deliv. 4 (2) 101-110 (2007).

52. Applications of carbon nanotubes in drug delivery. A. Bianco, K. Kostarelos, M. Prato. Curr. Opin. Chem. Biol. 9:674-679 (2005).

53. Aerogels: tailor-made carriers for immediate and prolonged drug release. I. Smirnova, S. Suttiruengwong, W. Arlt. Kona 23, 86-97 (2005).

\section{Reviews on Medical Imaging and Theranosties}

1. Biomedical applications of functionalized hollow mesoporous silica nanoparticles: focusing on molecular imaging. S. Shi, F. Chen, W. Cai. Nanomedicine 8(12) doi: 10.2217/nnm.13.177 (2013).

2. Progress on the multifunctional mesoporous silicabased nanotheranostics. J-L Shi, Y. Chen, H-R Chen. J. Inorganic Materials Vol 28 Iss 1. 1-11, (2013).

3. Nanostructured porous silicon in preclinical imaging: Moving from bench to bedside. H.A. Santos, L.M. Bimbo, B. Herranz. J. Mater. Res. (2012).

4. Multistage nanovectors: from concept to novel imaging contrast agents and therapeutics. B.Godin, E.Tasciotti, X.Liu, R.E.Serda, M.Ferrari. Acc. Chem. Res. 44(10) 979-989 (2011).

5. Multifunctional mesoporous silica nanocomposite nanoparticles for theranostic applications. J.E. Lee, N. Lee, T. Kim, J. Kim, T. Hyeon. Acc. Chem. Res. 44(10) 893-902 (2011).

6. Mechanized silica nanoparticles: a new frontier in theranostic nanomedicine. M.W. Ambrogio, C.R. Thomas, Y.L. Zhao, J.I. Zink, J.F. Stoddart. Acc Chem Res. 44(10) 903-13. doi: 10.1021/ar200018x. (2011).

7. Nanoscale metal organic frameworks: magnetic resonance imaging contrast agents and beyond. J.Della Rocca, W. Lin. Eur. J. Inorg. Chem. 24, 3725-3734 (2010).

\section{Reviews on cancer detection and therapy}

1. Porous silicon for cancer therapy: from fundamental research to the clinic. A. Tzur-Balter, G. Shtenberg, E. Segal. Reviews in Engineering DOI 10.1515/revce2015-0001, 1-16 (2015).

2. Targeted Treatment of Cancer with Nanotherapeutics Based on Mesoporous Silica Nanoparticles. N.Z. Knezevic, J-O. Durand. Chem. Plus. Chem. Vol 80. Issue 1. 26-36 (2015).

3. Smart multifunctional drug delivery towards anticancer therapy harmonized in mesoporous nanoparticles. S.M. Baek, R. Singh, D. Khanal, K.D. Patel, E-J. Lee, K.W. Leong, W. Chrzanowski, H-W Kim. Nanoscale, 7(34) 14191-14216, DOI. 10.1039/c5nr02730f. (2015).

4. Targeted treatment of cancer with nanotherapeutics based on mesoporous silica nanoparticles. N.Z. Knezevic, J.O. Durand. Chem Plus Chem 80(1) 26-36 (2015) 
5. Advances in mesoporous silica nanoparticles for targeted stimuli-responsive drug delivery. A. Baeza, M. Colilla, M. Vallet-Regi. Expert Opin. Drug Delivery 12(2) 319-337 (2015)

6. pH-responsive mesoporous silica nanoparticles employed in controlled drug delivery systems for cancer treatment. K.N. Yang, C.Q. Zhang, W. Wang, P.C. Wang, J.P. Zhou, X.J. Liang. Cancer Biol. Med. 11:3443 (2014).

7. Functionalization of Mesoporous Silica Nanoparticles for Targeting, Biocompatibility, Combined Cancer Therapies and Theragnosis. Y. Kuthati, P-J. Sung, C-F. Weng, C-Y Mou, C-H. Lee. J. Nanosci. Nanotechnol. Vol 13. $2399-2430$ (2013).

8. Non-metallic nanomaterials in cancer theranostics: a review of silica- and carbon-based drug delivery systems. Y-C. Chen, X-C. Huang, Y-L. Luo, YC. Chang, Y-Z. Hsieh, H-Y. Hsu. Sci. Technol. Adv. Mater. 14, 044407 (2013).

9. Nanoporous silicon as Drug Delivery Systems for Cancer Therapies. S.M. Haidary, E.P. Córcoles, N.K. Ali. J. of Nanomaterials. Vol 10. 1155. 503-830. (2012).

10. Hybrid nanoparticles for detection and treatment of cancer. M.J. Sailor, J.H. Park. Adv. Mater. 24(28) 37793802 (2012)

11. Nanoparticles in targeted cancer therapy: mesoporous silica nanoparticles entering preclinical development stage. J.M. Rosenholm, V. Mamaeva, C. Sahlgren, M. Linden. Nanomedicine (Lond) 7 (1). 11120 (2012).

12. Multifunctional mesoporous silica nanoparticles for combined therapeutic, diagnostic and targeted action in cancer treatment. J.M. Rosenholm, C. Sahlgren, M. Lindén. Curr. Drug Targets 12, 1166-1186 (2011).

13. Towards biocompatible nanovalves based on mesoporous silica nanoparticles. Y.W.Yang. Med.Chem.Commun. 2,1033-1049 (2011).

14. Opportunities and challenges for carbon-based nanomaterials for cancer therapy. A. Bianco, K. Kostarelos, M. Prato. Expert Opin. Drug Deliv. 5(3) 331-342 (2008).

\section{Reviews on Tissue Engineering \& Cell culture}

1. Bone Tissue engineering using silica-based mesoporous nanobiomaterials: Recent progress. N. Shadjou, M. Hasanzadeh. Mater. Sci. Eng C. 55. 401-409 (2015).
2. Development of nanomaterials for bone repair and regeneration. R.E. McMahon, L. Wang, R. Skoracki, A.B. Mathur. J. Biomed mater Res A Appl Biomater. 101(2) 387-97 (2013).

3. Nanoporous Aluminium Oxide Membranes as Cell Interfaces. D. Bruggemann. J. Nanomaterials 1-18, ArticleID 460870 (2013).

4. Carbon-based nanomaterials for tissue engineering. S.H. Ku, M. Lee, C.B. Park. Adv. Healthcare Mater. 2, 244-260 (2013).

5. Nanofibrous scaffolds for dental and craniofacial applications. M.J.Gupte, P.X.Ma. J.Dent.Res. 91(3) 227-234 (2012).

6. Growth factor delivery-based tissue engineering: general approaches and a review of recent developments. K. Lee, E.A. Silva, D.J. Mooney. J. R. Soc. Interface 8, 153-170 (2011).

7. Biomimetic nanofibrous scaffolds for bone tissue engineering. J.M.Holzwarth, P.X.Ma. Biomaterials 32(36) 9622-9629 (2011).

8. Sol-gel silica-based biomaterials and bone tissue regeneration. D.Arcos, M.Vallet-Regi. Acta Biomateriala 6(8) 2874-2888 (2010).

9. Cell culture on MEMS platforms: A Review. M. Ni, W.H. Tong, D. Choudhury, N.A.A. Rahim, C. Iliescu, H. Yu. Int. J. Mol. Sci. 10, 5411-5441 (2009).

10. Porous bioactive nanostructured scaffolds for bone regeneration: a sol-gel solution. O.Mahoney, J.R.Jones. Nanomedicine 3(2) 233-245 (2008).

11. Nanostructured mesoporous silicas for bone tissue regeneration. I. Izquierdo-Barba, M. Colilla, M. Vallet-Regi. J. Nanomaterials Vol 2008 1-14 Article ID 106970 (2008).

12. Methods for fabrication of nanoscale topography for tissue engineering scaffolds. J.J. Norman, T.A. Desai. Annuals of Biomedical Engineering Vol 34 No 1, 89101 (2006).

13. Electrospinning of polymeric nanofibers for tissue engineering applications: A Review. Q.P. Pham, U. Sharma, A.G. Mikos. Tissue Engineering Vol 12. No 5, 1197 (2006).

14. Synthetic biomaterials as instructive extracellular microenvironments for morphogenesis in tissue engineering. M.P Lutolf, J.A. Hubbell. Nature Biotechnology 23. 47-55 (2005).

15. Making tissue engineering scaffolds work. Review on the application of solid freeform fabrication technology to the production of tissue engineering scaffolds. E. Sachlos, J.T. Czernuszka. Euro. Cells and Mater. Vol 5. 29-40 (2003). 
16. Scaffolds in tissue engineering bone and cartilage. D.W. Hutmacher. Biomaterials 21, 2529-2543 (2000).

\section{Reviews on biofiltration, biomolecule immobilization \& bioanalysis}

1. Alginate / porous silica matrices for the encapsulation of living organisms: tunable properties for biosensors, modular bioreactors, and bioremediation devices. M. Perullini, M. Calcabrini, M. Jobbagy, S.A. Bilmes. Mesoporous Biomater. 2 : 3-12 (2015).

2. Optical sensing and analyte manipulation in solid state nanopores. T.Gilboa, A.Meller. Analyst. 140, 4733-4747 (2015)

3. A review on porous silicon based electrochemical biosensors: Beyond surface area enhancement factor. C. RoyChaudhuri. Sensors and Actuators B: 210, 310-323 (2015).

4. Advanced nanoporous materials for microgravimetric sensing to trace-level bio/chemical molecules. P. Xu, X. Lin, H. Yu, T. Xu. Sensors 14, 19023-19056 (2014).

5. Porous silicon chemical sensors and biosensors: A review. F.A. Harraz. Sensors. Actuators B: 202: 897912 (2014).

6. Enzymes immobilized in mesoporous silica: a physical-chemical perspective. N. Carlsson, $\mathrm{H}$. Gustafsson, C. thorn, L. Olsson, K. Holmberg, B. Akerman. Adv. Colloid Interface Sci. 205, 339-360 (2014).

7. Nanoporous anodic alumina: a versatile platform for optical biosensors. A. Santos, T. Kumeria, D. Losic. Materials 7, 4297-4320 (2014).

8. Solid-state and biological nanopore for real-time sensing of single chemical and sequencing of DNA. F.Haque, J.Li, H.C.Wu, X.J.Liang,P.Guo. Nano Today 8, 56-74 (2013).

9. Progress in enzyme immobilization in ordered mesoporous materials and related applications. Z. Zhou, M. Hartmann. Chem.Soc.Rev. 42(9) 3894-3912 (2013).

10. Single molecule sensing with solid-state nanopores. B.N.Miles, A.P.Ivanov, K.A.Wilson, F.Dogan, D.Japrung, J.B.Edel. Chem.Soc.Rev. 42,15-28 (2013).

11. Immobilization of enzymes on porous silicasbenefits and challenges. M.Hartmanm, X.Kostrov. Chem.Soc.Rev. 42, 6277-6289 (2013).

12. Immobilization of enzymes on mesoporous silicate materials. E.Magner. Chem.Soc.Rev. 42(15) 6213-6322 (2013).
13. Mesopore-assisted profiling strategies in clinical proteomics for drug/target discovery. R. Savino, R. Terracciano. Drug Discovery Today 17(3-4) 143-152 (2012)

14. Recent advances of mesoporous materials in sample preparation. L. Zhao, H. Qin, R. Wu, H Zou. J. Chromatography A 1228, 193-204 (2012).

15. Bioanalysis based on nanoporous materials. Z. Dai, H. Ju. Trends Analyt. Chem. 39, 149-162 (2012).

16. In mesopore protein digestion: a new forthcoming strategy in proteomics. R. Savino, F. Casadonte, R. Terracciano. Molecules 16(7) 5938-5962 (2011).

17. Biotechnical and other applications of nanoporous membranes. P. Stroeve, N. IIeri. Trends in Biotechnology TIBTEC-879, 1-8 (2011).

18. Biocatalysis with enzymes immobilized on mesoporous hosts: the status quo and future trends. M. Hartmann, D. Jung. J. Mater. Chem. 20, 844-857 (2010).

19. Nanoporous membranes for medical and biological applications. S.P. Adiga, C. Jin, L.A. Curtiss, N.A.Monteiro-Riviere, R.J.Nararyan. Rev.Nanomed.Nanobiotechnol. 1(5) 568-581 (2009).

20. Enzyme-functionalized mesoporous silica for bioanalytical applications. C. Ispas, I. Sokolov, S. Andreescu. Anal. Bioanal. Chem. 393(2) 543-554 (2009).

21. Mesoporous materials for encapsulating enzymes. C.T.Lee, T.S.Lin, C.Y.Mou. Nano Today 4, 165-179 (2009).

22. Nanoporous Materials for Biomedical Devices. S.P. Adiga, L.A. Curtiss, J.E. Elam, M.J. Pellin, C-C. Shih, C-M. Shih, S-J. Lin, y-Y. Su, S.D. Gittard, J. Zhang, R.J. Narayan. JOM 26-32 (2008).

23. Proteins in mesoporous silicates. S. Hudson, J. Cooney, E. Magner. Angew Chem. Int. Ed. Engl. 47(45) 8582-94 (2008).

24. Assemblies of biomaterials in mesoporous media. A.Vinu, M.Miyahara, K.Ariga. J. Nanosci. Nanotechnol. 6(6) 1510-1532 (2006).

25. Ordered mesoporous materials for bioadsorption and biocatalysis. M. Hartmann. Chem. Mater. 17(18) 4577-4593 (2005). 


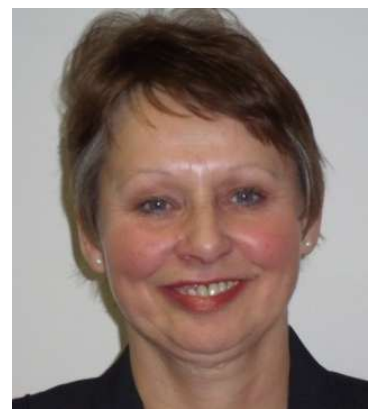

Elizabeth Kennedy Editorial Assistant

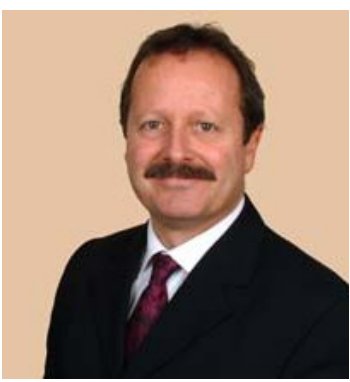

Leigh Canham Editor In Chief

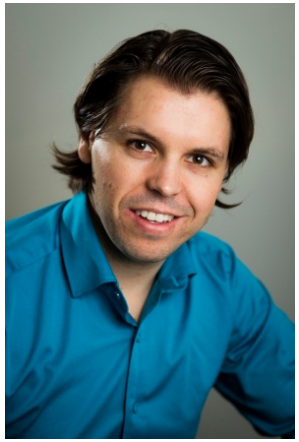

Hélder A. Santos

Journal Editor 